\title{
Guest editorial: Special issue on superlubricity
}

\author{
Ali ERDEMIR ${ }^{1}$, Jianbin LUO ${ }^{2}$ \\ ${ }^{I}$ Energy Systems Division, Argonne National Laboratory, Argonne, IL 60439, USA \\ ${ }^{2}$ State Key Laboratory of Tribology, Tsinghua University, Beijing 100084, China \\ Received: 14 June 2014 \\ (C) The author(s) 2014. This article is published with open access at Springerlink.com
}

Energy and material losses due to friction and wear in mechanical systems account for huge economic and environmental burdens. Approximately one-third of the world's primary energy consumption is attributed to friction; in addition, about $80 \%$ of the equipment failure is caused by wear in friction processes. Even relatively small improvements in the tribology of mechanical systems would reap enormous societal benefits.

Superlubricity is a state in which two contacting surfaces exhibit almost no resistance to sliding, and the friction force between the two sliding surfaces nearly vanishes. Improvement of superlubricity technology and our understanding of its mechanism play an important role for saving energy in industry as well as our daily life. Consequently, superlubricity has attracted a large amount of attention from researchers in many fields, which is leading to a revolution in engineering technology.

This Special Issue of Friction is intended to introduce readers to the exciting fields of superlubricity, covering not only the material types and fundamental mechanism of superlubricity but also its applications to industry. Review papers and research articles are included to demonstrate the breadth and the timeliness of the subject and to provide an opportunity for the publication of new findings. This issue contains nine invited papers by tribologists and scientists in materials science, physics, and chemistry, including

- Four review articles on the atomistics of superlubricity, superlubricity on the nanometer scale, principles and application to structural lubricity, and the superlubricity of diamond-like carbon (DLC) coatings;
- One research paper on the super-low friction of a-C:H and ta-C coatings;

- Two papers on the superlubricity of sapphire against ruby and sapphire against silicon nitride under phosphoric acid solution;

- One on experimental advances in superlubricity;

- One on surface passivation and boundary lubrication of self-mated tetrahedral amorphous carbon asperities.

The first paper, by Motohisa Hirano, reviews the history of research on the origin of friction, including the molecular theory of friction and the problem of energy dissipation in friction. Superlubricity is theoretically elaborated from the viewpoint of the atomistic origin of friction. In addition, the relation between friction and superlubricity is discussed in detail.

Ernst Meyer et al. review recent advances in superlubricity arising from experimental work and numeric simulations and discuss the factors that influence superlubricity on the atomic length scale. Specifically, they focus on structural lubricity, superlubricity at low normal forces, dynamic actuation of tip-sample contact resulting in superlubricity, and the thermal activation of friction. Also discussed are important solid superlubricity materials, such as $\mathrm{MoS}_{2}$, $\mathrm{Ti}_{3} \mathrm{SiC}_{2}$, multi-walled carbon nano-tubes, and polymers. Finally, the conditions for achieving superlubricity are explained by drawing upon the Prandtl-Tomlinson model and experimental results.

Nano-particle manipulation experiments, as described in the review by Dirk Dietzel et al., represent an opportunity to investigate the physical conditions and processes that are necessary to establish structural lubricity. Their review starts by introducing the 
limitations of standard atomic force microscopy (AFM) and possible strategies to circumvent them by pushing/pulling well-defined nano-objects with the same tip used for AFM imaging. In the rest of the manuscript the connection between interfacial friction and quantitative analysis in such manipulation experiments (power dissipation in the dynamic mode and lateral force in the contact mode) is discussed and exemplified by a series of key results on $\mathrm{Sb}$ nano-islands. Finally, this review provides a brief introduction to structural lubricity and a comparison with recently published measurements.

Ali Erdemir et al. review the superlubricity of DLC coatings by controlling the tribochemistry in the bulk material and at the surface. Hydrogen can be incorporated into bulk DLC or near surface regions during deposition or through hydrogen plasma treatment after the deposition. Hydrogen can also be fed into the sliding contact interfaces of DLCs during tribological testing. Due to favorable tribochemical interactions, these interfaces become very rich in hydrogen and, thus, provide super-low friction after a short run-in period. Regardless of the method used, when sliding surfaces of DLC films are enriched in hydrogen, they can provide super-low friction coefficients, as low as 0.001. Based on tribological and surface analytical findings, a mechanistic model is provided for the critical role of hydrogen in the superlubricity of DLC films.

Makoto Kano et al. have investigated the frictional characteristics of two types of DLC, a-C:H and ta-C, in the presence of 1-hexadecene and oleic acid. The ta-C can lead to the super-low regime with oleic acid, where the friction coefficient is below 0.01 , and the a-C:H is well lubricated by 1-hexadecen. The tribofilm chemistry and super-low friction mechanism with selected lubricants are elaborated by evaluating the friction of self-mated DLC and surface analyses.

The superlubricity behavior of sapphire against ruby under phosphoric acid lubrication was experimentally studied by Jinjin Li et al. in Jianbin Luo's group. They report that the load, sliding speed, and humidity of the test environment can affect superlubricity, and an ultra-low friction coefficient of 0.004 can be achieved under a high contact pressure (2.57 GPa). The excellent friction properties imply the potential application of phosphoric acid solution in the lubrication of sapphire bearings under high pressure.

The hydrodynamic effect on the superlubricity of phosphoric acid between silicon nitride and sapphire was investigated by Mingming Deng et al. in Jianbin Luo's group. The mechanism of superlubricity is revealed by direct observation of the contact area. The results of the friction coefficient versus sliding speed support the regime changing from boundary lubrication in the running-in period to elastohydrodynamic lubrication in the superlubricity period. One particularly interesting finding is the good agreement between the increase of contact area, concentration of phosphoric acid, and the achievement of superlubricity.

Superlubricity between incommensurate surfaces can provide the desired low-friction state essential for the function of small-scale machines and solid lubricants. Quanshui Zheng et al. discuss recent experimental observations of superlubricity ranging from the micrometer to centimeter scale in an ambient environment. They have developed a novel tribological technology based on a self-retracting motion phenomenon, and have observed the superlubricity phenomena at the microscale for graphite and at the macroscale for carbon nanotubes. Large-scale superlubricity was found to persist in ambient conditions and at high speeds (up to $25 \mathrm{~m} / \mathrm{s}$ for highly ordered pyrolytic graphite).

The closing paper by Pedro A. Romero et al. presents a comprehensive look, based upon molecular dynamics simulations, at the plastic deformation and phase transformation during shearing of ta-C films at the single asperity scale with or without lubricants. They systematically studied the composition and microstructure of tribomaterial under different conditions, finding that $\mathrm{H}$-passivation and hexadecane molecules could reduce the ta- $\mathrm{C}$ rehybridization and lead to formation of an $\alpha-\mathrm{C}: \mathrm{H}$ tribomaterial. They have gained a better understanding for the role of surface passivation and lubrication in atomic-scale friction. 